City University of New York (CUNY) CUNY Academic Works

\title{
Assessment of Creative Thinking in an Introductory Robotics Course Using Final Project
}

Lili Ma

CUNY New York City College of Technology

Jose Reyes Alamo

CUNY New York City College of Technology

Yu Wang

CUNY New York City College of Technology

\section{How does access to this work benefit you? Let us know!}

More information about this work at: https://academicworks.cuny.edu/ny_pubs/769

Discover additional works at: https://academicworks.cuny.edu

This work is made publicly available by the City University of New York (CUNY).

Contact: AcademicWorks@cuny.edu 


\section{Assessment of Creative Thinking in an Introductory Robotics Course Using Final Project}

\section{Dr. Lili Ma, New York City College of Technology}

Professor Lili Ma received her Ph.D. in Electrical Engineering from Utah State University focusing on autonomous ground vehicles. After that she did three-year post-doctoral training at Virginia Tech working with autonomous aerial vehicles. Prior to joining the Computer Engineering Technology (CET) department at City Tech in fall 2016, she taught at Wentworth Institute of Technology for many years. Professor Ma's research areas include autonomous mobile robots, vision-based control, visual servoing, visual tracking, coordinated control, and sensing \& perception techniques.

\section{Dr. José M. Reyes Álamo, New York City College of Technology}

José M. Reyes Álamo did his undergraduate studies in Computer Science at the University of Puerto Rico at Bayamón. After graduation he worked in the industry but his strong passion for education and research brought him back to school a year later. He went to Iowa State University (ISU) to pursue his Ph.D. in Computer Science. During his time at ISU he worked in the Smart Home Lab researching topics in Software Engineering, Smart Homes, Pervasive Computing, Formal Methods, and Wireless Sensors. He published several papers and completed his dissertation entitled "A framework for safe composition of heterogeneous SOA services in a pervasive computing environment with resource constraints". In ISU he was also involved in several other activities like organizing the GMAP Symposium, participating in student organizations, and working as research assistant. After graduation he worked for a trading software company in New York City but once again his love for academics brought him back, this time as a faculty in Computer Engineering Technology in the CUNY - New York City College of Technology.

\section{Dr. Yu Wang, New York City College of Technology}

Dr. Yu Wang received her Ph.D. degree in Electrical Engineering from the Graduate Center of the City University of New York in 2009. Currently she is a professor in the Department of Computer Engineering Technology at New York City College of Technology. Her primary area of interest includes engineering education, formal methods for modeling real-time systems, digital design, Agile testing, embedded systems, and network protocols. 


\title{
Assessment of Creative Thinking in an Introduction Robotic Course using Final Project
}

\begin{abstract}
This study describes development of an assignment (the final project) used for assessment of creative thinking in an undergraduate robotic course. Robotics inherently demands creativity, requiring exploration and investigation among several different methods to eventually generate a satisfactory solution. Assessment of students' creative thinking fits naturally into our robotic course, which has a 2.5-hour lab session each week for students to work on physical/simulated robots. Students are asked to complete three projects over one semester. This paper describes the re-design and fine-tuning of the final project such that assessment of creative thinking can be incorporated into this robotic course as a regular component.

While many courses offer opportunities for students to work on projects, feedback were usually provided at the stage of final products. The process of creative thinking was instead insufficiently assessed. The objective of this study is to design a well-structured robotic-specific assignment that emphasizes and promotes creative thinking throughout the semester. Five weeks were allocated in fall 2020 for the final project. Constructive feedback was provided to students weekly, which in turn improved the overall quality of the final products.

Instead of using one single topic/task in the final project, several candidate topics are provided. Each group, typically consisting of one or two students, are allowed to select the one that they are most interested in pursuing. Each candidate topic is associated a "risk index", indicating how challenging this option can be as perceived by the instructor, and thus the level of risk that students need to take to complete this particular task. A lower risk index corresponds to a less risk-taking decision. Further, for each candidate topic, hints and suggestions of possibly more than one method are provided. Students' ability of embracing contradictions is evaluated by if students have considered and/or implemented various methods. A higher assessment score is given to groups who have investigated and compared multiple different methods (at least more than one method) before settling down on one solution. Acquiring competencies is evaluated based on the cumulative/overall capability that each student has acquired and demonstrated from the beginning till the end of the semester. Solving problems is evaluated by the completeness of the task, being definitely satisfactory, satisfactory, acceptable, or unacceptable. Finally, evaluation of innovative thinking is based on if each group has proposed and implemented their own solutions other than those suggested.
\end{abstract}

In summary, five out of six performance criteria from the Creative Thinking Value Rubric were assessed in fall 2020 using the final project of the robotic course offered. Creative Thinking Value Rubric was used for the performance criteria. This paper presents three carefully-designed candidate topics for the final project, together with the collected assessment results. We believe that by including this assessment as a regular course component, students' creative thinking capability will be enhanced to better prepare them for future careers.

\section{Introduction}

The course CET 4952: Robotics Technology in the department of Computer Engineering Technology at CUNY-New York City College of Technology is offered as a technical elective to its senior students. In addition to introducing fundamental subjects in both Autonomous Mobile Robot [1] and Robotic 
Manipulator [2], another goal is to prepare students with necessary knowledge and skills for robotic programming and design. The course is structured to have a 2.5-hour lecture session and a 2.5-hour lab session each week. When teaching onsite the school (i.e., in-person), students were given physical robots for implementation of the algorithms discussed during lectures. When access to laboratory facilities was impossible under e-learning (for example, during the COVID-19 pandemic), computer-based simulation projects of similar complexities were used to address the same topics.

Creative thinking is an important skill needed for modern workplace in engineering and technology fields [3]. Instructors in higher education consider various ways of integrating creativity and innovation into the courses they teach to better prepare students for their future careers [4]. Study shows that between the two commonly-used approaches that enhance creativity, i.e., teaching vs. practice creativity, stimulating creativity by practices is considered to be more effective [5]. In addition, many professors used projectbased learning $[6,7]$ when trying to foster creativity in their courses $[8,9]$.

Due to robotics' multi-disciplinary nature, we think that inspiration of creative thinking fits naturally in the robotic course. Particularly, the lab sessions provide an opportunity for instructors to include and encourage creative thinking practices. Over years, project-based learning $[6,7,10]$ has been adopted and students are asked to perform three projects in the lab session. The first two projects are used to guide students through fundamental and commonly-addressed algorithms. Inquiry-based learning [11, 12], a highly self-directed approach of learning and discovering through experiments or observation, is used in the final project, where students apply acquired skills to solve some open-ended questions independently (with the least amount of help from the instructor). The combination of project-based learning and inquiry learning prepares students with basic knowledge and skills (through projects 1 and 2), as well as exposing them (through the final project) to open questions that promote creative thinking.

\section{Assessment of Creative Thinking via Final Project}

Studies report that robotics has a positive impact on students' development of creative thinking, along with many other important skills needed for modern workplace, including, for example decision making, problem solving, communication, and team working, to name but a few [13, 14]. Finding a solution to an open-ended robotic control task independently involves several key elements of creative thinking, including acquiring knowledge, innovative thinking, embracing contradictions, solving problems, and risk-taking.

Acquiring proper background knowledge is a vital part of being a critical thinker. These fundamental yet essential knowledge \& skills are introduced in both lectures and the first two projects (projects 1 and 2). The third project provides students with an opportunity to practice their creative thinking skills by solving open-ended questions, and thus is used as the assessment tool for creative thinking. The nature of being open-ended implies that multiple solutions might exist for a given problem. Students thus need to explore among various methods (i.e., embrace contradictions) to find the most appropriate approach, and/or to come up with a completely new way (i.e., applying innovative thinking) to solve the problem (i.e., problem solving). Further, our teaching methodology of providing only supervision (instead of detailed instructions) pushes the students to consider the risk factor as well.

\section{Candidate Topics for Final Project}

We believe that students will spend more time and efforts in the final project if the task/topic is interesting to them. Thus, instead of assigning the same topic for all students, three carefully-designed topics were developed in fall 2020, covering the three important themes of this robotic course (i.e., autonomous mobile 
robots, robotic manipulator, and advanced robotic control, respectively). In other words, the final project includes three candidate topics (or options), among which students were allowed to choose one. These three options are briefly described below.

\section{Option 1: Map Building \& Path Planning of an Autonomous Mobile Robot}

This option is an extension of Project 1 on Autonomous Mobile Robots. Project 1 is regarding path planning and waypoint navigation of a robot working in a completely known environment, i.e., all objects and their positions are known beforehand. The robot will find an obstacle-free path from its current position to a specified goal location and then follow the path, as shown in Fig. 1 (c) and (d). As an extension, the objects' positions are assumed to be unknown. This requires the robot to first detect the positions of the unknown objects using its onboard range sensors; determine the number of objects by applying certain clustering algorithm; and then find the area covered by each detected object [as shown Fig.1 (a), (b)], before applying the path planning algorithm.

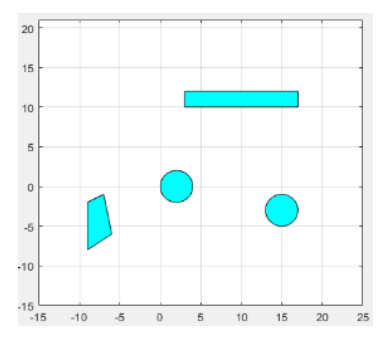

(a) Unknown Environment

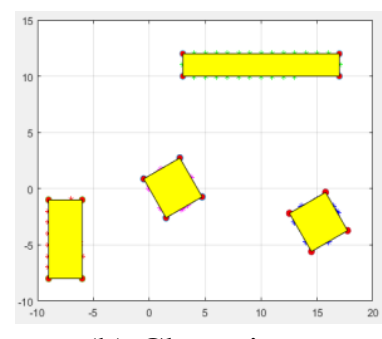

(b) Clustering

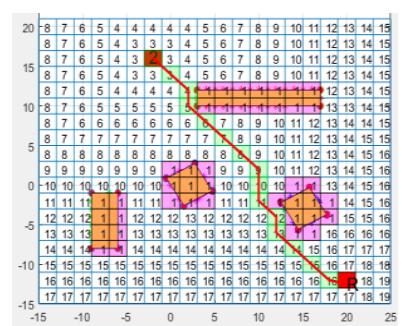

(c) Path Planning

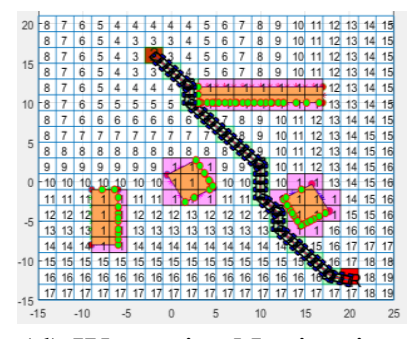

(d) Waypoint Navigation

Figure 1: Path planning of an autonomous mobile robot in an unknown environment.

\section{Option 2: Trajectory Generation of a 4-DOF SCARA Robot}

This option is similar to Project 2 on Robotic Manipulators. Given a Selective Compliant Articulated Robot for Assembly (SCARA) as shown in Fig. 2, the task is to perform trajectory generation of the robot's endeffector in both the 3D space and the joint space for a given set of 3D "Via-points". The project was implemented using Peter Corke's MATLAB Robotic Toolbox [15]. As an alternative design method, students were suggested to implement their own forward and inverse kinematics sub-functions, instead of using functions provided by the toolbox.

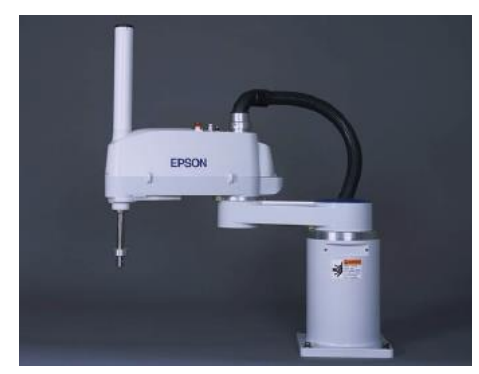

(a) SCARA Robot

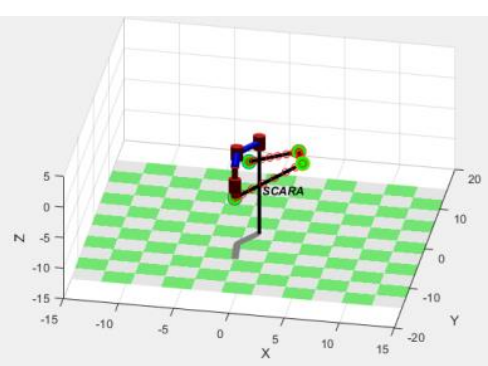

(b) 3D Space

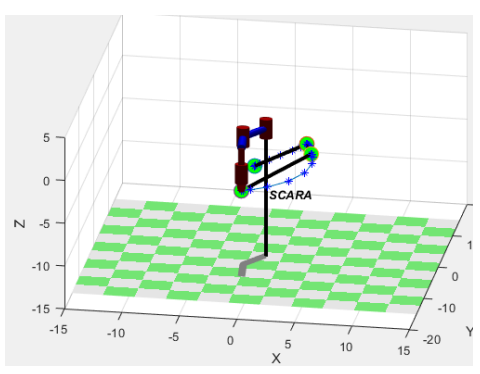

(c) Joint Space

Figure 2: Trajectory generation and inverse kinematics of a SCARA robot.

Option 3: Sensing, Perception, and Control of a Robot using the MATLAB-ROS Platform

This option is to perform sensing, perception, and control of a simulated robot using the MATLAB-ROSGazebo package. Robotic Operating System (ROS, https://www.ros.org/) is an open source, Linux-based robotics development system that supports research and development robots. Recently, interests arose from 
educators to introduce ROS to undergraduate robotic curriculum/courses, as well as promoting undergraduate research investigations $[16,17,18,19]$. Mathworks' ROS Toolbox, which provides a library of functions that exchange data with ROS-enabled physical or simulated robots, make programming much easier and thus manageable for undergraduate students. Figure 3 shows a simulated Gazebo Playground environment, laser and image collected by the robot's onboard sensors, and feature extraction of a blue object.

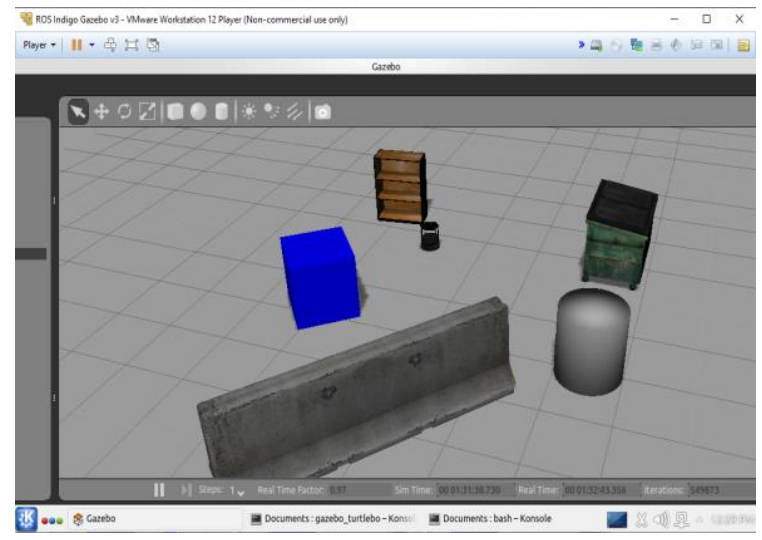

(a) Gazebo Playground Environment

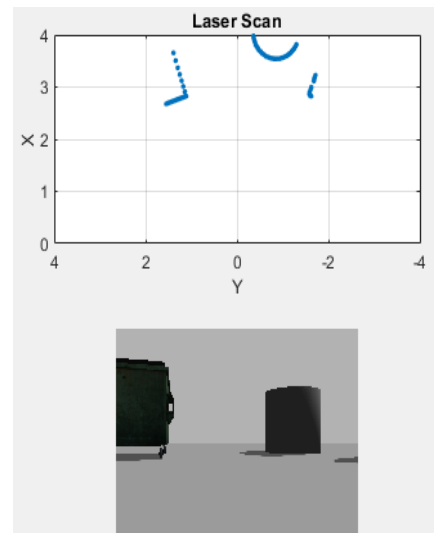

(b) Laser \& Image

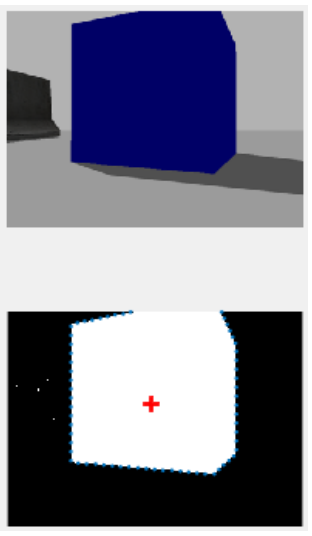

(c) Image Processing

Figure 3: Sensing, perception, and control of a robot using the MATLAB-ROS platform.

In fall 2020, the MATLAB-ROS platform was introduced to the students using five examples, showing students how to collect laser data and image sequences, perform simple image processing and obstacle avoidance, and send control commands to the robot. Students were then asked to proceed further and explore on their own. They were allowed to define their own control objective, as long as the following three elements are conducted: (1) collection of onboard sensor data (laser and/or images); (2) processing of these data; and (3) usage of the processed information to control the motion \& behavior of the robot. Students' work in fall 2020 included visual servoing to an object, controlling the distance between a target and the robot, and usage of an external device (an Arduino board) to control the velocity of the robot.

\section{Assessment Method}

Among the six criteria of assessing creative thinking, the following five were evaluated using the final project in fall 2020 under e-learning with a student body of 21 students. Though students were allowed to form groups with another student (a maximum of 2 students in one group), 7 students chose to work on their own. The rest of 14 students formed 7 groups of 2 students per group. Evaluation was mainly based on group performance, except for "Acquiring Competence".

Acquiring Competences: Evaluation was based on performance of each student. Before the final project, students had completed Projects 1 and 2. Students' cumulative grades of Projects 1 and 2, were used as the initial score of this criterion. During the final project, any coding/debugging questions/help requested by the students resulted in certain points off (since this shows that students have not yet grasped the basic skills). Eventually, this indicator shows how capable and independent each student is, revealing the readiness and competence acquired by the student for creative thinking practices. 
Risk-Taking: Each of the three options of the final project was assigned a risk indicator by the instructor. This indicator shows how challenging each option can be as perceived by the instructor. Clearly, students who choose more complicated options take higher risk, and thus have exhibited higher-level risk-taking capabilities. Specifically, the risk indicators of the three options are:

- Option 1: risk indicator is 3

- Option 2: risk indicator is 2

- Option 3: risk indicator is 4

Embracing Contradictions (Alternative Design): This indicator shows if the students take/seek an alternative design method.

- Score-1: if the group didn't even consider an alternative design method.

- Score-2: if the group considered an alternative design but didn't try to implement it.

- Score-3: if the group considered an alternative design method, tried to implement it, and the result was reasonably satisfactory.

- Score-4: if the group considered an alternative designed method, tried to implement it, and the results was successful.

Innovative Thinking (Creative Design): This indicator shows if students have implemented any new ideas (or any of their own ideas) in the design.

- Score-1 or 2: Students didn't apply any of their own/new ideas.

- Score-3: Students included some of their own/new ideas into the project, but the task was achieved with the help of the instructor.

- Score-4: Students included some of their own/new ideas into the project and they completely everything on their own.

Solving Problems (Completeness): This indicator shows if the task was successfully completed. Score 4, 3, 2 and 1 are given based on the completeness of the project, corresponding to being definitely satisfactory, satisfactory, acceptable, or unacceptable.

\section{Assessment Results}

Assessment of students' creative thinking capabilities was performed in fall 2020 under e-learning with a student body of 21 students. The assessment results are presented in Fig. 4. Setting 70\% as the target for being "Proficient and/or Satisfactory", it can be seen that "Acquiring Competence", "Solving Problems", and "Embracing Contradictions" all meet the target reasonably well. It was observed that students spent great amounts of efforts in the final project. Several students performed even better than what had been demonstrated earlier in the semester.

The criterion of "Risk-Taking" (71\%) barely meets the target of $70 \%$ and "Innovative Thinking" (67\%) is below the target. This assessment identifies these two areas as the areas that deserve attention for future improvement. 


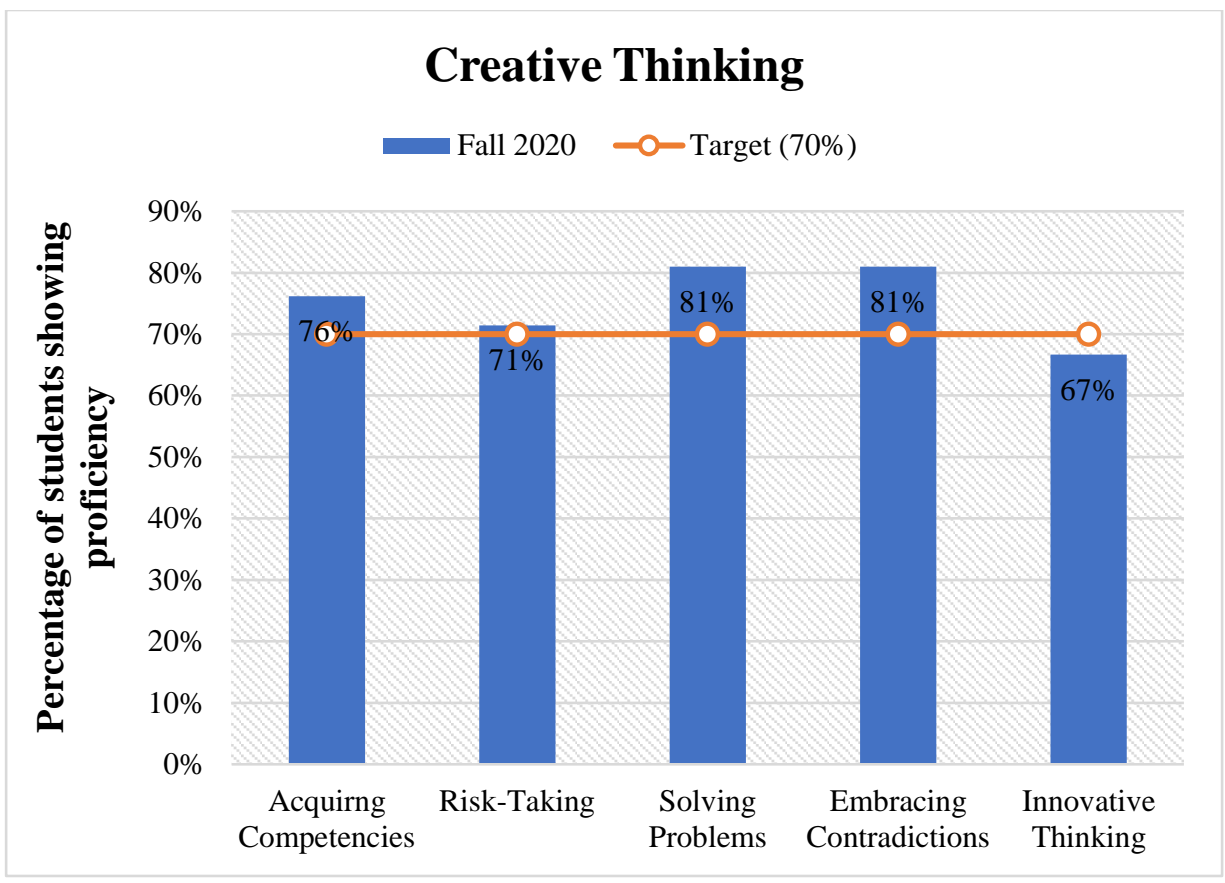

Figure 4: Assessment of students' creative thinking capabilities in fall 2020.

\section{Conclusions and Future Work}

Creative thinking is an important skill needed for modern workplace in engineering and technology fields. Our approach to foster and then assesses creative thinking in an undergraduate robotic course is via the format of using two projects to prepare students with the necessary competence and then providing them with an opportunity (the final project) to practice creative thinking by tackling an open-ended question. Particularly, the first two projects intend to prepare students with fundamental knowledge and skills needed for robotic design and programming. The final project expects students to explore and apply ideas of their own when finding a solution for an open-ended robotic control task. This approach was validated in fall 2020 as an effective method to promote and assess creative thinking. We plan to include this assessment work as a regular course component in the future.

The assessment in fall 2020 helps to identify "Risk-Taking" and "Innovative Thinking" as the two areas that deserve attention for future improvement. For "Risk-Taking", 6 out of 21 students selected Option 2, which is the easiest among all three options. For future improvement, this option will be replaced by a more challenging version. This will push students to go beyond their comfort zone, thus helps to improve their risk-taking capabilities. Adding more options and allowing a maximum number of groups to choose the same option may help to improve risk-taking as well. Students will then have fewer other classmates to count on, requiring them to take more risks themselves.

For "Innovative Thinking", 7 out of 21 students didn't provide a solution other than those directed by the instructor. In other words, these solutions lack creative thinking from their own. For future improvement, we will encourage students to form a group with other students. By interacting with each other in a regular basis, listening to other people's perspectives, and brain-storming (of problems, progress, and plan), students may learn to reconstruct their own/new ideas and develop creative-thinking habits.

In the future, we will also apply the assessment method described in this paper to other design courses to foster, promote, and evaluate students' creative thinking skills \& capabilities. 


\section{References}

[1] R. Siegwart, I. Nourbakhsh and D. Scaramuzza, Introduction to Autonomous Mobile Robot, MIT Press, 2004.

[2] M. W. Spong, S. Hutchinson and M. Vidyasagar, Robot Modeling and Control, Wiley, 2020.

[3] A. Bielefeldt and A. Morse, "Teaching and Assessment of Innovation and Creativity in Civil Engineering: Why? How? Now!," in Annual Conference and Exposition, 2019.

[4] J. Pappas and E. Pappas, "Creative Thinking, Creative Problem Solving, And Inventive Design In The Engineering Curriculum: A Review," in Annual Conference and Exposition, 2003.

[5] A. Martin-Erro, M. M. Espinosa and M. Dominguez, "Creativity and Engineering Education: A Survey of Approaches and Current State," in International Technology, Education and Development Conference, 2016.

[6] E. H. Fini, F. Awadallah, M. M. Parast and T. Abu-Lebdeh, "The Impact of Project-Based Learning on Improving Student Learning Outcomes of Sustainability Concepts in Transportation Engineering Courses," European Journal of Engineering Education, vol. 43, no. 3, 2018.

[7] A. Shekar, "Project Based Learning in Engineering Design Education: Sharing Best Practices," in ASEE Annual Conference \& Education, Indianapolis, IN, 2014.

[8] N. Wijayati, W. Sumarni and S. Supanti, "Improving Student Creative Thinking Skills Through Project Based Learning," in UNNES International Conference on Research Innovation and Commercialization, 2019.

[9] S. Y. Chen, C. F. Lai and Y. H. Lai, "Effect of Project-Based Learning on Development of Students' Ceative Thinking," International Journal of Electrical Engineering \& Education, 2019.

[10] L. L. Wu, E. Zhu, C. Callaghan, D. Irwin, D. Reinsdorf, V. Swanson, A. Zwirn and D. Reinkensmeyer, "Rapidly Converting a Project-Based Engineering Experience for Remote Learning: Successes and Limitations of Using Experimental Kits and a Multiplayer Online Game," Advances in Engineering Education, vol. 8, no. 4, 2020.

[11] H. Altin and M. Pedaste, "Learning Approaches to Applying Robotics in Science Education," Journal of Baltic Science Education, vol. 12, no. 3, 2013.

[12] T. De Jong and V. Joolingen, "Scientific Discovery Learning with Computer Simulations of Conceptual Domains," Review of Educational Research, vol. 69, no. 2, pp. 179-201, 1998.

[13] N. Eteokleous, E. Nisiforou and C. Christodoulou, "Creativity Thinking Skills Promoted Through Educational Robotics," in Advances in Intelligent Systems and Computing, 2020.

[14] D. Alimisis, "Educational Robotics: Open Questions and New Challenges," Themes in Science and Technology Education, vol. 6, no. 1, pp. 63-71, 2013.

[15] P. Corke, "Robotics Toolbox," 2020. [Online]. Available: https://petercorke.com/toolboxes/roboticstoolbox/.

[16] S. Andrew, J. Forsyth and C. Korpela, "Project Based Learning Using the Robotic Operating System (ROS) for Undergraduate Research Applications," in ASEE Annual Conferences and Exposition, 2017.

[17] W. Lehman and M. Hayder, "Introducing Kinematics with Robot Operating System (ROS)," in ASEE Annual Conference and Exposition, 2015.

[18] K. Khan, "ROS-Based Control of a Manipulator Arm for Balancing a Ball on a Plate," in ASEE Annual Conferences and Exposition, 2017.

[19] S. Wilkerson, J. Forsyth, C. Sperbeck, M. Jones and P. Lynn, "A Student Project using Robotic Operating System (ROS) for Undergraduate Research," in ASEE Annual Conference and Exposition, 2017. 